LAWRENCE LIVERMORE NATIONAL LABORATORY

\title{
Large Diffractive Optics for GEO-Based Earth Surveillance
}

R. Hyde

September 11, 2003 
This document was prepared as an account of work sponsored by an agency of the United States Government. Neither the United States Government nor the University of California nor any of their employees, makes any warranty, express or implied, or assumes any legal liability or responsibility for the accuracy, completeness, or usefulness of any information, apparatus, product, or process disclosed, or represents that its use would not infringe privately owned rights. Reference herein to any specific commercial product, process, or service by trade name, trademark, manufacturer, or otherwise, does not necessarily constitute or imply its endorsement, recommendation, or favoring by the United States Government or the University of California. The views and opinions of authors expressed herein do not necessarily state or reflect those of the United States Government or the University of California, and shall not be used for advertising or product endorsement purposes.

This work was performed under the auspices of the U.S. Department of Energy by University of California, Lawrence Livermore National Laboratory under Contract W-7405-Eng-48. 


\section{Large Diffractive Optics for GEO-Based Earth Surveillance}

The natural vantage point for performing Earth-centric operations from space is geosynchronous orbit (GEO); a platform there moves at the same rate as the Earth's surface, so appears to continually "hover" over a fixed site on the Earth. Unlike spacecraft in other orbits, which rapidly fly-over targets, a GEO-based platform remains in-position all the time. In order to insure continual access to sites using low earth orbit (LEO) platforms, one needs a large enough constellation $(\sim 50)$ of spacecraft so that one is always overhead; in contrast, a single GEO platform provides continuous coverage over sites throughout Euro-Asia.

This permanent coverage comes, unfortunately, with a stiff price-tag; geosynchronous orbit is $36,000 \mathrm{~km}$ high, so space platforms there must operate at ranges roughly 100 times greater than ones located in LEO. For optical-based applications, this extreme range is difficult to deal with; for surveillance the price is a 100-fold loss of resolution, for laser weapons it is a 10,000-fold loss in flux-on-target. These huge performance penalties are almost always unacceptable, preventing us from successfully using GEO-based platforms. In practice, we are forced to either settle for brief, infrequent access to targets, or, if we demand continuous coverage, to invest in large, many-satellite, constellations.

There is, fortunately, a way to use GEO-based optical platforms without incurring the huge, range-dependent, performance penalties; one must 'simply' use bigger optics. As long as the aperture of a platform's optics increases as much as its operating range, then its performance (resolution and/or flux) does not suffer; the price for operating from GEO is 'simply' 100-fold larger optics.

This is, of course, a very stiff price; while meter-class optics may suffice for many low-earth-orbit applications, 100 meter apertures are needed in order to achieve similar performance from GEO. Since even the largest Earth-based telescope is only 10 meters in diameter, building ten-fold larger ones for GEO applications (let alone delivering and operating them there) presents major difficulties. However, since the challenges of fielding large platforms in GEO are matched by the benefits of continuous coverage, we propose a program to develop such optical platforms.

In this section, we will examine a particular form of large aperture optic, using a flat diffractive lens instead of the more conventional curved reflectors considered elsewhere in this report. We will discuss both the development of this type of large aperture optics, as well as the steps necessary to use it for GEO-based Earth surveillance. In a later section of this report we will discuss another use for large diffractive optics, their application for global-reach laser weapons. 


\section{Technical Discussion of Large Diffractive Lenses}

Building and fielding very large space optics is difficult for two reasons, their precision and their size. The applications we're interested in require diffraction-limited optical performance, to achieve this, light must be controlled to sub-wavelength levels of precision. For example, the surface of a visible-wavelength reflector must be accurately positioned to $\sim 25 \mathrm{~nm}$ in order to achieve high performance operation. It is difficult enough to reach this level of precision over few meter apertures, doing so over a 100 meter span is very much more challenging. The second difficulty, of course, is that these very large telescopes have to be used in space; they have to be lightweight enough and compact enough to be delivered to GEO. On Earth, optical precision has historically been attained by using stiff, rigid structures. For 100 meter-sized GEO applications, however, this approach is completely infeasible, not only would the telescope be too heavy to launch, but also it could not be packaged compactly enough to fit within a booster.

These twin difficulties have long been appreciated, and much work has been, and is being, done to address them. For example, modern large telescopes, such as the 10 meter Keck ones, no longer rely on global stiffness to hold optical precisions over their apertures; instead they use adaptive optics. Here, sensors measure the optical precision of the telescope, and actuators are actively controlled via feedback loops to adjust the position of the telescope's surface (either at its primary aperture or at secondary locations). This is an extremely powerful technique, but not a panacea when aiming for 100 meter scale space-based optics. The limitation concerns the number of surface locations that must be actively controlled. If the optical aperture is lightweight and floppy, then control points must be closely spaced, and many will be required to cover a 100 meter aperture $\left(10 \mathrm{~cm}\right.$ spacing requires $10^{6}$ control points); if the surface is stiffened to allow wider-spaced control points, then the mass required for a 100 meter aperture rapidly becomes prohibitive.

Reducing aperture weight is, naturally, a major thrust of current R\&D efforts; but this work is very far removed from the levels required for 100 meter-scale optics. For instance the goal for the Jack Webb Space Telescope is (for launch in 2011) to reduce areal mass from the Hubble's $200 \mathrm{~kg} / \mathrm{m}^{2}$ down to $15 \mathrm{~kg} / \mathrm{m}^{2}$. This target is, of course, far too high for 100 meter-scale optics, even a $1 \mathrm{~kg} / \mathrm{m}^{2}$ value would result in a 10 ton aperture. The size of optics needed for GEO-based applications drives one inexorably towards much lighter surfaces; the price, of course, will be the size and complexity of the adaptive optics system needed to insure optical precision.

Telescopes based upon diffractive lenses, instead of mirrors, are an approach to deal with both the mass-and-launchability and the optical-precision problems facing large reflective telescopes. For basic physics reasons, thin-film lenses require much looser optical tolerances than reflectors, greatly enhancing the near-term practicality of fielding large, 100 meter-scale, GEO-based optics. The other advantage of diffractive lenses is that they can be made from flat, thin-film materials; thinness allows low weight, while flatness greatly simplifies launch packaging and in-space mechanical support. 
These pervasive advantages arise from the fundamental properties of diffractive lenses. Diffractive optics work by applying small, local corrections to a light beam, instead of a large, global correction. This is illustrated below; conventional optics focus light by applying a smooth phase change of many-waves, while diffractive optics apply a large number of small, single-wave corrections.

\section{Conventional - - Diffractive}

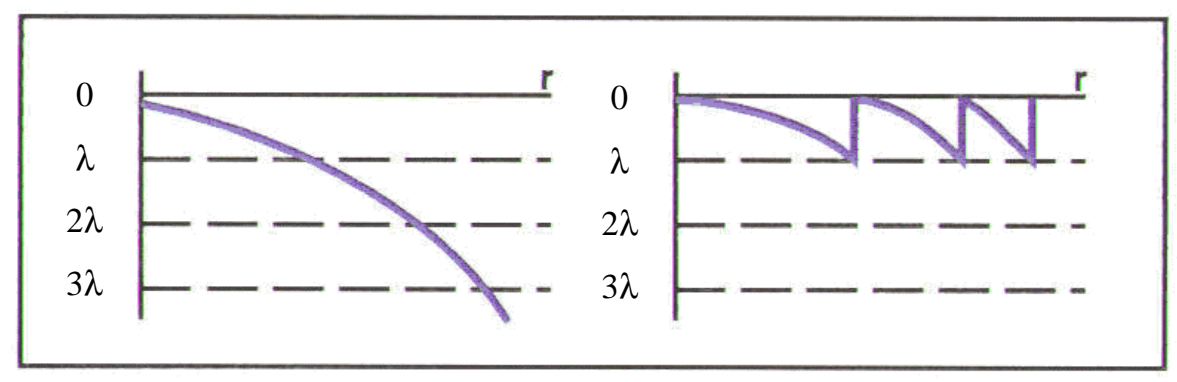

Physically, a diffractive optic (either a reflector or a lens) can be implemented by a series of shallow, closely spaced, grooves on the surface of an aperture. Because these grooves are shallow ( $\sim 1 \mu \mathrm{m}$ for a visible-light lens) the material holding them can be thin and lightweight; for instance $a_{-}$mil plastic film has a mass of only $0.02 \mathrm{~kg} / \mathrm{m}^{2}$.

While diffractive optics can be used for either thin lenses or reflectors, choosing lenses leads to huge tolerance advantages. The reason, illustrated below, is fundamental; light passes through a lens, but is reversed by a reflector.
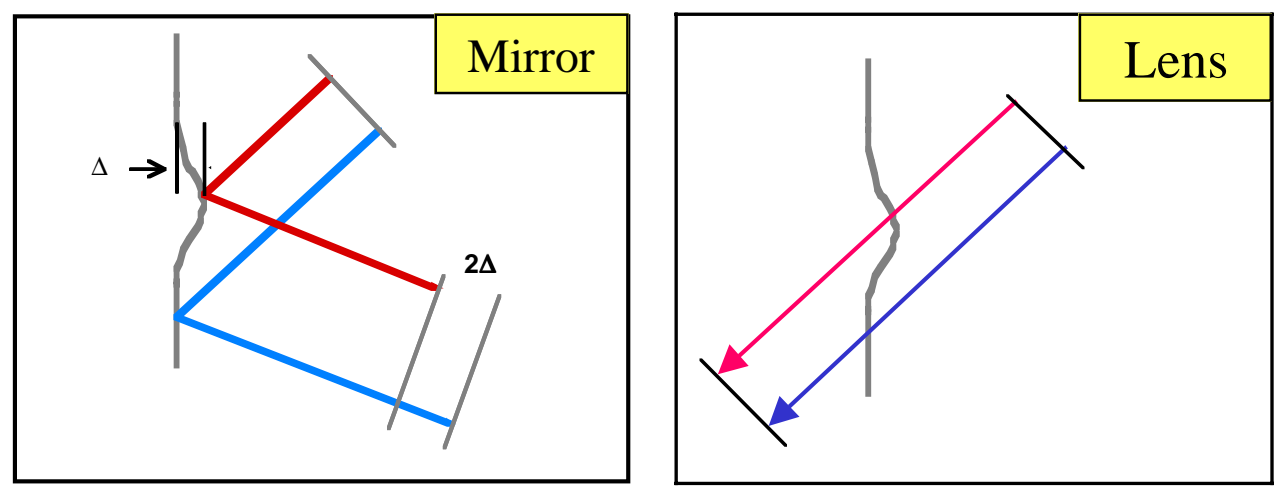

Because mirrors reflect light, the optical-pathlength errors associated with their surface ripples are doubled. In contrast, pathlength errors for a thin transmissive film are cancelled-out, as light first arrives-at and then departs-from surface ripples.

In actual lenses, this pathlength cancellation is not perfect since (because the lens must focus light) the incoming and outgoing rays are not traveling in quite the same directions. If the light is bent through angle $\theta$, then the pathlength error in a mirror is amplified (above the physical shape error) by a factor of $(1+\cos \theta)$, while that in a thin lens is reduced by $(1-\cos \theta)$. Lenses therefore have a $(1+\cos \theta) /(1-\cos \theta)$ tolerance 
advantage over mirrors. By making the lens weak, i.e., by keeping $\theta$ small, this tolerance gain becomes huge, scaling as $4 / \theta^{2}$, i.e., as $(4 \mathrm{~F} / \mathrm{D})^{2}$. A thin lens having an $\mathrm{f}$-number (F/D) of 50 can tolerate 40,000 times greater floppiness than a similar mirror. This raises visible-light tolerances from $\sim 25$ nanometers up to a millimeter, a tremendous practical advantage when trying to field a large, lightweight aperture in space.

The other major advantage of diffractive lenses is that they are easier to field in space than are large reflective apertures. Mass, of course, is critical when trying to implement a 100 meter space aperture; both membrane reflectors and diffractive lenses utilize thin polymer films and hence offer the potential of low mass apertures, even at the 100 meter scale. Large apertures, however, require supporting structure as well as the optical surface itself; these support structures can easily dominate the overall mass of the aperture. Here, because diffractive lenses are easier to hold in shape than are reflectors, they allow considerably simpler, and lighter optical apertures.

Part of the fielding advantages of diffractive lenses will, of course, stem from their much looser figure requirements. However, an even greater fielding advantage comes from the fact that diffractive lenses are flat membranes, not curved ones as reflectors must be. The optical focal power of a reflector comes from its global shape, it must be curved in order to focus light. For a diffractive lens, however, focusing comes from the spacing of its grooves; the surface these lie on can be, and generally is, designed to be flat. The ability to use flat membranes brings with it two major benefits. The first is that flat membranes can be held taut purely by edge forces applied at the boundaries of the membrane. However, in order to keep a curved membrane taut, one must also apply precise side forces over the entire surface area of the film. Membrane reflectors require some means (usually either gas pressure or electrostatic) of applying such side forces everywhere over their surface in order to hold them into a precise curve; diffractive lenses are flat and need no such areal forces.

The other benefit of flat membranes is that they are much easier to package than curved films are. The large aperture optics needed for GEO applications are much bigger than the payload volume in launch vehicles; in addition to being light enough for launch, they must also be tightly packaged in order to fit within the boosters. One way of doing so, is to launch the aperture in small pieces, and then reassemble it in space. While possible, this places an additional major developmental effort (learning how to do large, high-precision space assembly) in series with the effort and risks of developing large aperture optics; if at all possible, launching a preassembled membrane is a much preferable approach.

In principle, one should be able to take advantage of the flexibility of thin-films and simply wad-up the membrane into a tight bundle. The reason such a straightforward approach isn't feasible, is that it causes creases, which are the bane of membrane optics (lenses or mirrors). The problem is that creases globally (not just locally) distort the membrane, and that, once set, it is very hard to 'pull-out' creases to the levels needed for optical precision. So, in order to launch large membranes, we must have a way to tightly package them without creasing. Here, flat membranes have a fundamental advantage over 
curved ones; flat membranes can be rolled (without any creases) or carefully folded (with creases only at specific, protectable, places). In contrast, curved membranes cannot be rolled or folded without developing creases throughout their area. Because diffractive lenses are flat, they avoid this problem.

Diffractive lenses do, however, have their own set of challenges and difficulties; solving these problems is a major focus of the developmental program proposed here. One of the leading challenges to making and fielding diffractive lenses is, as for reflectors, optical precision. While the precision requirements are different and smaller than for reflectors, they still dominate the challenges of fielding diffractive lenses.

A diffractive lens has three different types of precision requirements; we'll illustrate them by describing the levels needed to achieve $\lambda / 10$ quality in the visible. The first need, is that the thin-film comprising the lens must have uniform thickness, to a level of $\sim 100 \mathrm{~nm}$. While this is comparable to the $25 \mathrm{~nm}$ surface accuracy required of reflectors, the crucial difference is that for lenses this is a fabrication challenge, not a space-fielding one; for reflectors it is both. Once a lens membrane has been made on the ground to $100 \mathrm{~nm}$ smoothness, nothing that happens to it in space will change the thickness significantly; for lenses this is a build-then-forget requirement.

The second precision requirement is the most challenging one; the grooves responsible for diffractive focusing must be placed correctly to an accuracy (for an f/50 lens) of $\sim 5 \mu \mathrm{m}$. This is 200 -fold looser than the $25 \mathrm{~nm}$ reflector requirement, and also different since it is an in-plane requirement (the stiff direction for taut membranes), not an out-of-plane one (the floppy direction). Nonetheless, achieving this precision will be difficult, particularly since it involves both fabrication and fielding challenges. Unlike the smoothing requirement, the job will not be finished once the grooves have been formed in the right location; they have to remain properly placed after they're in space.

There are, unfortunately, several space-fielding effects which can expand or contract the membrane by more than $5 \mu \mathrm{m}$. Creases are a major concern here, this is why crease-free packaging is essential. The tension applied to keep the membrane taut will stretch it, but proper geometry and pre-compensation will handle these effects. The most challenging in-space distortions are thermal ones; temperature changes will expand or contract the membrane. Fortunately, the largest effects (due to orbital Sun-angle changes) are spatially uniform and do not degrade the lens's focal quality. The danger comes from smaller non-uniform thermal gradients (due, for instance, to shadowing or reflections from the membrane's support structure). These must be dealt with by proper design of the lens and its support structure, as well as by using low thermal-expansion (low CTE) lens materials.

The final precision requirement for diffractive lenses is on the film's out-of-plane positioning. This is, of course, the most challenging requirement for a reflector (particularly since it is the floppy direction for membranes), but is where diffractive lenses have a huge advantage over reflectors (in this case, 40,000-fold), raising tolerances 
from $25 \mathrm{~nm}$ up to $1 \mathrm{~mm}$. While some care must be taken to keep the lens membrane flat, doing so is not a major challenge.

One operational challenge to using large diffractive lenses is that they have long focal lengths. The optical tolerance requirements for these lenses improve with f-number (linearly for in-plane requirements and quadratically for out-of-plane ones) so there is a strong incentive for fielding slow, high f-number, systems. The combination of a large fnumber and a large diameter means that the focal length of these diffractive lenses is generally several kilometers. This makes it impractical to field simple, single vehicle, platforms for GEO-based applications; systems must contain two separated spacecraft, one being the large lens, and the other the secondary collection/transmission optics. In order to operate as a high-precision optical system, these two spacecraft must cooperate, holding position with each other. Neither the precision nor propulsive requirements for this stationkeeping are particularly severe, but the need to operate a dual-vehicle platform will certainly be an operational complication.

In addition to the technical challenges of building and fielding large diffractive lenses, their novelty poses significant programmatic concerns. Compared to the widespread capabilities and experience-base in building and operating reflective telescopes, there is very little existing infrastructure to support large diffractive optics. While the largest diffractive lenses are comparably sized to reflectors (5 meters compared to 10), there are far fewer organizations with experience in building or operating them. In developing large diffractive optics, their inherent technical advantages will be countered by the extra risk associated with their novelty.

The effort to develop large space-based, diffractive lenses can be broken up into 6 separate areas: Material, Fabrication, Configuration \& Support, Delivery, Space Operations, and Optical Control.

\section{Lens Material}

Selecting the proper material for a space-based diffractive lens is similar to, but more difficult-than, choosing one for a membrane reflector. As with a reflector, the lens material must be thin, lightweight, reasonably strong, and space-stable. To be useful for a diffractive lens, however, the material must also be transparent, and should have a low CTE (thermal expansion coefficient).

All of these criteria can be satisfied by certain inorganic materials, such as silica, glass, sapphire, etc. Such materials have a number of outstanding properties (e.g., mechanical stiffness, space stability, optical transmission), and have long been used for both conventional and diffractive optics; the recent LLNL 5 meter diffractive lens was built from $0.7 \mathrm{~mm}$ thick glass sheets. There are, however, two drawbacks to use of inorganics for future, very large, space lenses. One concern is that such lenses will need very thin, $10-50 \mu \mathrm{m}$, materials; it is considerably more challenging to form large freestanding inorganic membranes, than it is with polymer materials. The second concern is with the brittleness of thin inorganic materials; handling, packaging, launching, and 
deploying such films will be much more challenging than with flexible materials such as polymer films. These two difficulties are not insurmountable, but are sufficiently troublesome, that, if feasible, polymers are preferable.

Polymer films have two significant advantages compared to inorganics, they are readily available in thin $(10-50 \mu \mathrm{m})$ films, and they are not brittle. Countering this, unfortunately, are two major drawbacks; most polymers degrade rapidly in the UV and radiation environment of space, and most have quite high thermal expansion coefficients. Unless these difficulties can be resolved, polymers are unsuitable for this application. Fortunately, there are many polymers to choose from, and some do appear to have the required properties.

The most attractive candidates appear to be members of the polyimide family of polymers. Polyimides are a high temperature polymer, with a long history of successful use in space; both their mechanical properties and transmittance holdup well in the vacuum, UV, and radiation of space. The other attraction of polyimides is that there are many different ones to choose from. Polyimides are a binary polymer, made by combining diamine and dianhydride monomers; mixing different diamines and dianhydrides allows great flexibility in tailoring material properties such as transparency and thermal expansion. This flexibility can be illustrated by comparing 3 flavors of polyimide. Kapton is the most common polyimide, with a long flight history; unfortunately it is effectively opaque in the blue-end of the spectrum, and has a large CTE. During the 1980s NASA developed a transparent polyimide called CP1, which is now available commercially; $\mathrm{CP} 1$ has some successful spaceflight experience, but as with most plastics, features a high CTE. The polyimides which appear most promising for this application are based on the TFDB diamine; they are fairly transparent (closer to CP1 than to Kapton) and can be tailored to have a near-zero CTE. One challenge to use of TFDB-based polyimides is that (unlike Kapton or CP1) there is, as yet, no commercial source for large films. The other concern is simply that there is no spaceflight experience with these polyimides; while it is expected that they will share the space-stability of other polyimides, this has not yet been demonstrated.

\section{Lens Fabrication}

Turning a thin film of material into a diffractive lens requires two things; the film must be smoothed to a uniform thickness, and it must be patterned with shallow, precisely located, surface grooves.

The first issue, is whether these tasks should be done on the full-scale monolithic membrane, or on smaller segments. While there are attractions to full-sized patterning, these are outweighed by practical difficulties. It is easier to acquire or develop tooling to handle small sections of material, and doing so decouples fabrication development from the sizing of the operational lens. Furthermore, one may not want to build a monolithic lens; the difficulties of handling and packaging a full-sized lens are severe, and may be best dealt with by launching the lens in separate pieces. In any event, the few $\mu \mathrm{m}$

precision needed to align and join together pre-fabricated lens segments (on the ground or 
in space) is not a major challenge; segmented fabrication is clearly warranted. The next question, is what type of segments should be made; the basic choice is between tiles and strips. This choice will depend on the material used, the details of the patterning and smoothing processes, the launch packaging, and the lens configuration. Because fewer pieces must be handled, strips (handled and packaged in rolls) are generally preferred, but, depending on the above circumstances, tiles may turn out best.

The primary fabrication issues, the smoothing and patterning procedures, depend intimately on the materials being used.

If the lens is made from a polymer film (such as a polyimide), then the initial material is most likely supplied as a rolled-up strip. It would clearly be preferable if this was formed, as part of the manufacturing process, with the required uniformity. Unfortunately, the precision we require $(\sim 100 \mathrm{~nm})$ is tighter than current commercial tolerances. There seems no inherent reason that films with the necessary precision cannot be made, but inducing a supplier to do so will likely be expensive and technically risky. The more prudent approach is to buy the best available film, and then post-process it to deliver the required uniformity. The most straightforward smoothing process currently seems to be a closed-loop variant of laser ablation, a technique discussed below for applying diffractive patterns to the lens membrane.

Laser ablation is a process in which an intense laser pulse is applied to a surface. The uppermost material absorbs the light, is strongly heated, vaporized, and blown-off the surface. By varying the flux or number of pulses, the depth of ablation can be accurately controlled. This is a well developed process, and is routinely and commercially used to apply detailed surface patterns to polymer films, including polyimide ones. Several companies build and sell laser ablation stations, including rollto-roll ones; with modest customization these machines are quite capable of patterning polymer-film diffractive lenses with the precision and throughput needed for our applications.

Generally this processing is done in an open-loop mask-driven method; a mask is made defining the desired spatial pattern, the laser is focused through the mask onto the target film, and material is ablated to the required depth. This approach is very well suited to applying the regular grooves needed for a diffractive lens. It can also, however, be adapted to perform variable patterning in order to smooth as-purchased film. Here, as the film is transported through the ablation machine, it is first examined interferometrically to determine the local thickness profile. This is deconvolved into a set of simple, standardized profiles (such as polynomial or sinusoidal shapes); these patterns have been prewritten onto portions of a correction mask, mounted in the machine. The correct sections of mask are exposed to the appropriate number of laser pulses, and the measured thickness profile is ablated away in a hands-off, closed-loop process. Accordingly, laser ablation should be capable of both smoothing and patterning polymer films. We have already used laser ablation to ablate sophisticated, 16 level, lens patterns into both Kapton and CP1 films, so the basic feasibility of this patterning process has been verified. 
There is another, high-throughput commercial process which might be suitable for patterning polymer lasers. This involves heating the polymer, softening it, and then mechanically forming it into the proper shape. A number of such techniques exist, ranging from direct extrusion through a properly shaped die, to embossing from a patterned roll onto a blank polymer film. These methods are currently used to make diffractive optics such as holographic security films, microlenses, etc. There is very little doubt that this type of process can be used to apply lens patterns to polymer films for our applications; however, there are concerns. The first is that this approach is best suited for patterning the lens, not smoothing it; if laser ablation is needed for smoothing, it might as well also be used for the simpler patterning step. The second concern is with achieving the global precision needed for the groove locations; these methods heat and soften the lens film and then physical contact it; laser ablation, in contrast involves no contact or bulk film heating. For these reasons, ablation appears to be a more promising technique. However, the embossing-type methods are also worth pursuing; especially since it is conceivable that high-enough-precision extrusion methods could allow direct production of smoothed, patterned films.

Different patterning and smoothing processes are called for if the lens is made from inorganic materials such as glass or silica. While we have purchased and made optics from thin $(300-700 \mu \mathrm{m})$ glass sheets in the past, the thicknesses and formation processes are not suitable for the $10-50 \mu \mathrm{m}$ films desired for large aperture lenses. It appears that thin films such as these are best made by direct growth processes (such as sputtering). Large, multi-meter wide sputtering machines are used commercially to apply inorganic coatings to long strips of material (e.g., window glass). It seems likely that such machines could be adapted to grow thin inorganic films on sacrificial substrates (e.g., polyimide film); an added benefit is that this process might be precise and uniform enough to not require subsequent smoothing.

Many processes exist to pattern (and smooth if necessary) inorganic sheets. As an example, consider lithography; this is, of course, very well developed for semiconductor patterning. LLNL routinely performs large, meter-scale, lithography to make diffractive optics; for example, their recent 5 meter diffractive lens was patterned with this process. Lithographic patterning can be straightforwardly adapted to a continuous linear process for high-throughput patterning of long lens strips. Smoothing is a less regular process, but requires much less narrow features; high-rate processes such as MRF polishing or spatially controlled thermal etching are under development, and should be capable of the closed-loop smoothing of inorganic sheets.

\section{Lens Configuration \& Support}

In order to be useful in space as an optical aperture, the thin-film diffractive lens must be accurately held in place. There are many ways in which this can be done, but a successful design must be lightweight, easily erected, capable of holding the lens material taut with optical precision, and also to position and steer it. We will discuss several ways of supporting diffractive lenses, based upon two very different lens configurations. 
The illustration below shows that diffractive lenses can focus light either with circular, radially symmetric grooves, or with orthogonal, linear grooves. The first, circular, approach is a direct 2-D lens, while the second acts as two crossed cylindrical lenses, each focusing light in a single direction. There is, of course, no need for either type of lens to be restricted to a specific shape; while the natural shape for a circularly grooved lens is circular, and that for a crossed linear lens is square, the shapes and patterns can be mixed at will.

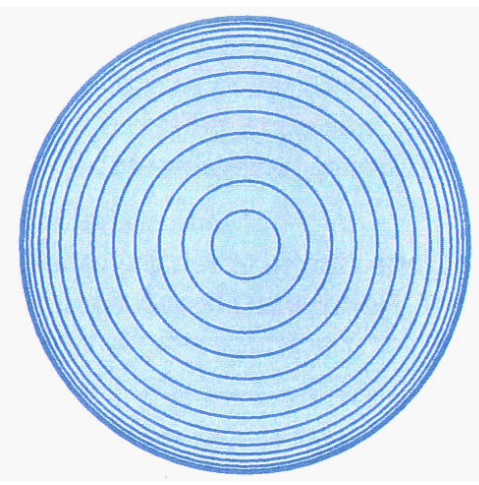

Circular

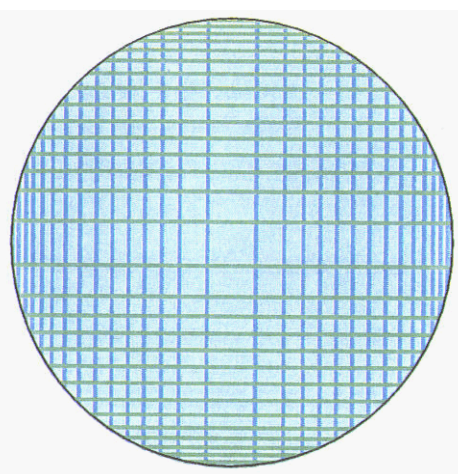

Linear

The primary requirement for the support structure of a large diffractive lens is to hold the thin-film lens taut, flat, and (most critically) to maintain the lateral precision $(\sim 5$ $\mu \mathrm{m})$ needed for accurate focusing. The biggest challenge to maintaining lateral precision is non-uniform thermal distortions. Since the lens is flat, and is heated by a uniform plane wave source (i.e., the Sun), the lens temperature should be the constant across its surface, and hence not disrupt focal quality. Non-uniform distortions (the only optically damaging ones) can, however, be introduced by external agents; the chief potential culprit being the lens's support structure. If the support structure itself distorts thermally (relative to the lens membrane), it can introduce distortions from the boundary where it grips the lens. Even if these direct effects are avoided by careful design, shadows or reflections from the structure can nonuniformly heat the membrane, indirectly distorting it.

The most straightforward structural approach to support a flat lens, is to pull it taut from the rim, like a drumhead. To avoid thermally disturbing the membrane, the force can be applied by an external structure which never crosses the lens aperture. As long as the structure's thermal expansion is matched to that of the lens material, and properly compliant (possibly controllable) mounts are used, such a structure should not feed distortions into the lens. When the Sun angle is close to the plane of the lens, the exterior structure will cast shadows onto the lens, disrupting its performance, but by mounting the structure sufficiently outboard of the lens, these outages can be minimized. External rims provide a convenient, low-risk way to support diffractive lenses, but will present significant mass, packaging, and deployment challenges for large, 100 meterscale, systems. 
The simplest way to avoid thermal distortions from the support structure is to not have one at all. Because the lens is simply a flat membrane, we can use centrifugal force to keep it taut; this Circular Spinner approach is illustrated below. The in-plane tension needed to keep the lens flat and taut is provided by slow, $\sim 1 \mathrm{rpm}$, axial rotation. The stretching induced by this rotation is small and predictable; the groove locations are set to be correct when spinning, not when built.

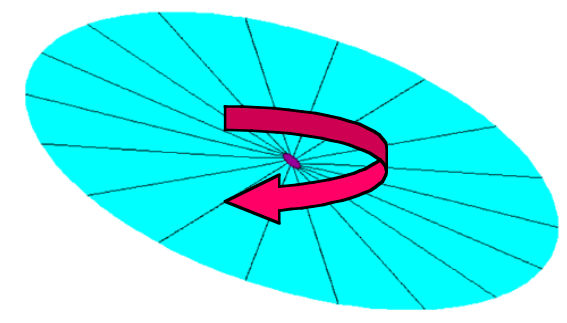

Circular Spinner

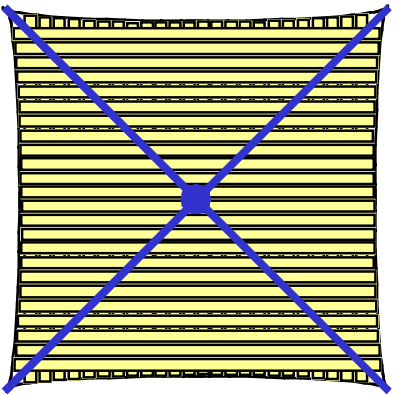

Square Ribbon

The biggest potential drawback of spinning, is that it makes the lens gyrostable, and hence hard to point; this could be a severe problem because earth-looking GEO lenses have to be rotated $360^{\circ}$ each day. This challenge is solved by placing a counterrotating gyrowheel at the center of the lens. This small, rapidly-rotating gyrowheel cancels the angular momentum of the large, slowly-spinning membrane; the full system has no angular momentum and can thus be steered at will. In fact, the gyrowheel provides a convenient way to controllably swivel the lens, both for general earth-pointing during its orbit, and for aiming at particular sites on the earth. This steering is done by slightly cocking the gyrowheel within its housing, thereby applying counter-torques to the lens, repointing it; this is the same principle used in a conventional control moment gyro.

Because the thin-film lens is a large, floppy object, the central torques used to swivel it will create deflections; the lens will no longer be flat. Fortunately, lenses are quite insensitive to such out-of-plane deflections; calculations show that although a swiveling lens is not physically flat, it does remain "optically" flat. The use of rotation, rather than an external rim, also eliminates the packaging and deployment concerns of such a large structure. The central hardbody is small and compact, and its gyrowheel can be gradually spun-up to slowly and controllably deploy the lens.

While the Central Spinner design is the 'cleanest' way to support a diffractive lens it does have some practical drawbacks. The first is programmatic; the nation has considerable experience with using truss-like structures to deploy and support things in space, but little with rotation. A system with two major novelties (diffractive lens and rotation) is much more programmatically risky than if only one 'miracle' is required. The other concern is technical; crease-free packaging of large monolithic films is a very hard thing to do. 
The Square Ribbon lens design (shown above) is an attempt to bypass these two concerns. It uses a truss to deploy and support the lens, and replaces the monolithic membrane with separate linear strips. Obviously the use of linear strips is well matched to the crossed cylindrical lens approach to focussing, each physical strip contains a set of linear grooves, unchanged along the length of the strip. The packaging advantages of separate strips are obvious, they can be handled, launched, and deployed as individual, meter-width, rolls; there is no need to handle or package a large monolithic membrane. The linear strips also make thermal distortions much easier to deal with; because distortions along the length of a strip have no optical effect, thermal strains only accumulate over the much shorter width of the strips. This $(\sim 100$-fold) advantage can be exploited to ease the CTE requirements on the lens material, and/or to allow use of simple, centrally deployed truss structures, rather than more complex, external rim ones.

There are two other practical advantages to using the Square Ribbon approach, with its separate linear strips. The first, is that simple 1D control of the strip attachment points allows the lens to be isolated from the effects of any truss distortions. The other advantage is programmatic; modularity bounds risk. Failures of individual strips are permissible, failure of a monolithic membrane is not.

\section{Lens Delivery}

To be useful, these large diffractive lenses have to be successfully delivered to space. While lunar materials may eventually be available for space applications, for the meantime, large lenses will have to be launched from Earth. In order to do this, both their mass and stowed volume must be tightly constrained.

Our current (and only planned) stable of launch vehicles, (e.g., the Shuttle and the Atlas V) can only launch 20 tons of payload into LEO. Existing upper stages could be used to deliver about 6 tons of this up to GEO, but by either using separately launched chemical stages or advanced electric-propulsion ones, the full 20 might be delivered. If all of this mass was devoted to the optical surface of a 100 meter aperture, it could have a $2 \mathrm{~kg} / \mathrm{m}^{2}$ specific mass. In practice, of course, the optical-surface can only be allotted a small fraction of the total mass of an optical platform; structure, propulsion, and application systems will dominate the mass budget. Because of this, plus the desire to not require advanced staging capabilities, the actual mass density available for 100 meterscale lenses is $\sim 0.05-0.10 \mathrm{~kg} / \mathrm{m}^{2}$. While these values are very far below current reflector values, they are not challenging for diffractive lenses; a straightforward 1 mil plastic membrane has a mass of only $0.04 \mathrm{~kg} / \mathrm{m}^{2}$. Mass will always be a concern for large diffractive lens platforms, but this is more a challenge for the structure and auxiliary systems than for the lens itself.

Packing the lens into a limited launch volume is the major challenge involved in delivering 100 meter-scale lenses into space. Obviously these lenses are far too big to fit directly into the booster, they have to be tightly stowed. The challenge here, is not the actual volume of the lens material (a 100 meter, 1 mil thick, film uses only $0.25 \mathrm{~m}^{3}$ ), it's the need to stow the lens without breaking or creasing it. 
The most direct way to stow the lens is to do so as separate panels, stacked on top of each other. This has a number of compelling advantages. The first, is its simplicity; no complex folding or rolling techniques have to be developed and demonstrated. A second advantage is its packaging density; a tight stack wastes no packing volume; most other approaches rapidly devour the 'ideally available' packing volume with gaps between layers of stowed material. A final advantage, related to the above ones, is that densepacks are well suited to surviving the launch process itself. Launch payloads are subjected to both steady and vibrational loads; holding delicate objects firmly in place, so they can't jerk around, is key to their survival. Insuring tight confinement (and then verifying it experimentally) is difficult for complex packaging schemes, but can be done quickly and easily for dense-packed films. This may become a compelling justification for the separate panel approach if thin, brittle materials such as glass sheets are chosen for the lens.

The drawback to separate panels is, of course, that they have to be assembled together in space. The precision required $(\sim 5 \mu \mathrm{m})$ is challenging, but not severely so. It is well within the state-of-the-art if global metrology is used for the alignment. Multipanel diffractive lenses have also been successfully assembled using purely local alignment. Here, each panel is registered with its neighbors via border fiducials. Because errors statistically accumulate, the individual alignments have to be more precise $(\sim 0.25$ $\mu \mathrm{m})$, but the overall assembly process is simpler because the alignments are local and there is no need for global metrology.

Since the precision requirements for assembling a large lens out of separate panels appear quite achievable; the bigger concern is likely to be the programmatic risks of remote, automated, assembly. Technically, this appears straightforward; it is a highly modular, repetitive operation, requiring the sequential alignment and attachment of $\sim 10,000$ meter-sized lens segments. A space-assembly robot can be designed for this task, and can be space tested with full-sized lens panels well before launch of the full lens. As in other cases, the largest drawback to this approach is that it requires two new developments (diffractive lens and space assembly) to succeed together. Countering this, is that the package and assembly of separate panels does appear technically straightforward, and that most other approaches to packaging large lenses carry their own major risks.

If possible, it would be preferable to avoid space assembly, and to deliver monolithic lenses monolithically, preassembled on the ground. Doing so, requires very careful packaging, so that the lens can be stowed and launched without breaking or creasing. The best way to do this, would be by 1D rolling, placing a long, rug-like roll, length-wise into the booster. Unfortunately, boosters are far too short for this approach to be used with 100 meter-sized lenses; 2D packaging is required.

The problem with 2D stowage of large membranes is the tight bends required for dense packaging; glass breaks and polymers crease. The lens's inability to recover from these effects is what prevents us from simply wadding-up a thin lens, since sharp folds 
will occur throughout the surface. Controlled folding schemes, in which fold locations occur at carefully preplanned locations, are much more promising. Here, the line folds can be protected (by joints in glass or crease-limiting rods in plastic), and the nodes where folds intersect can be dealt with by holes in the material. Newspaper-like folding is not attractive because it features thick, multi-layer folds, but more sophisticated origami techniques have been used successfully (e.g., for LLNL's 72 piece, 5 meter glass lens) The trouble with folding, is simply that a 100 meter lens requires many folds, which nest and overlay in complex fashions. When combined with the real-world complication that folds/hinges have finite thickness, it will be very challenging to devise a folding scheme to pack and protect a 100 meter-scale lens for launch. Doing so, and then verifying it, will constitute a major programmatic risk.

An approach which appears more attractive for monolithic lenses than pure folding, is a combined fold-then-roll method. Here, the lens membrane is first Z-folded in one dimension, using joints or crease-limiting rods at the folds. Then the long, multilayer, sandwich is rolled (over as gentle a radius as possible) in the second direction. Despite its attractions, there are concerns with this approach; the roll-bend interactions, the membrane deployment, and the difficulty of using this packaging method with a preattached membrane/support-structure combination, will all present challenges.

The Square Ribbon lens design offers an intermediate launch packaging scenario, between the separate-panel and the monolithic-membrane extremes. The significant virtue here, is that the lens material is in the form of separate strips, each full-length, but with manageable, meter-scale widths. These strips can be packaged in rolls, since the individual rolls are small enough to fit easily within the launch volume. Roll-packaging is, of course, the technique used routinely throughout industry for crease-free handling and protection of large thin films. It offers an attractive, and verifiable, way to package

and protect lens membranes for delivery to space. Once in space, the deployment and assembly of the strips is more straightforward than for separated panels; the rolls can be preattached to their support truss before launch, and the strips deployed from their rolls like window-shades.

\section{Lens Operation in Space}

The deployed lens has to function as a separate spacecraft. It hence requires auxiliary, non-optical systems to supply command capability, power, propulsion, and attitude control. Fortunately, there is nothing particularly challenging about these tasks or unique about their incorporation into a large diffractive lens.

One factor which simplifies the lens's auxiliary operations is that it functions cooperatively with a secondary platform; this is either the eyepiece in an imaging system or the laser in a weapon. Some of the lens vehicle's responsibilities, such as command capability, power supply, and relative-positioning propulsion can be handled by the secondary platform. 
The command system for the optical platform can clearly be situated in the secondary vehicle, and signals can be broadcast to the neighboring lens as required. The lens needs a power supply only to handle functions such as propulsion, attitude control, and, depending upon the design, gyro friction or active alignment of lens strips. A 10,000

$\mathrm{m}^{2}$ lens aperture presents ample area to mount thin-film solar arrays to generate the power for these systems. Alternatively, the neighboring secondary platform, whose power requirements will likely dwarf those of the lens, can use microwaves to transmit a small fraction of its power across to the nearby lens.

The lens will need its own propulsion and attitude control system. However, most of the system's propulsion needs will be required for the inter-vehicle stationkeeping, both to prevent the two vehicles from drifting apart, and to swing their pointing LOS between specific targets on earth; orbital maintenance needs are much less. The stationkeeping portion of the propulsive load can be handled either by the secondary platform or the lens, whichever is most convenient. The advantage of using the lens is that it will probably be the lighter of the two vehicles; the advantages of the secondary are that it is the source of power and has less vibration constraints. Whichever vehicle performs the maneuvers, the overall $\Delta \mathrm{V}$ requirements are large, about $5 \mathrm{~km} / \mathrm{s}$ per year for anti-drift control alone; but acceleration levels are small. Because of these characteristics, propulsion should be performed by electrical engines, not chemical ones; this increases electrical requirements, but drastically reduces propellant ones.

The lens will have to be swiveled frequently to point at desired targets. However, these requirements are not severe. The largest angular motion, the $360^{\circ}$ per day rotation to track the earth, is steady and needs no external torque. The frequent small maneuvers to shift between close-by targets do not require any lens swiveling, as they will fall within the large, few degree, FOV of such lenses. The only time the lens must be actively swiveled, is when one must shift between relatively distant targets. This motion can be provided either by gyroscopic or propulsive systems. The former is clearly preferred for a Circular Spinner type of lens, while either could be used for nonrotating designs such as a Square Ribbon lens.

\section{Optical Control}

One of the most crucial advantages of diffractive lenses is that they allow much looser tolerances than do reflectors. It is tempting to fully exploit this feature, and to field passively precise telescopes. While this remains a desirable goal, it does impose programmatic risk; what happens if the lens, once fielded, is not precise enough for high quality imaging? Accordingly, it is desirable to incorporate adaptive optical correction capability within the system; a belt-plus-suspenders approach.

There are several ways to actively correct errors in a large diffractive telescope. One, which has already been alluded to for a Square Ribbon lens, is inside the lens itself, by controlling the location where strips attach to the truss. The goal of this control is simply to correct the effect of truss errors; as truss distortions move the strip attachment points, we actively shift them back to their proper location. Because the strips are 
insensitive to out-of-plane and to longitudinal errors, each attachment point needs only 1D control of its sideways position. This control problem appears manageable; a typical lens will have about 100 strips in each direction, so we must control the linear location of 400 points, to accuracies of $\sim 5 \mu \mathrm{m}$. The required positioning information can either be provided by a global metrology system, or locally using neighbor-to-neighbor measurements.

Control of strip attachment-points can remove the effect of truss errors, but we may also need to correct errors caused within the lens membrane itself, due to either fabrication errors (film uniformity or groove patterning) or in-space ones (stationkeeping, thermal bowing, or vibrations). As with reflective telescopes, the preferred place to correct for such errors is within the optics of the secondary platform, rather than at the large lens. These matters will be discussed later, for the different types of secondary platforms.

\section{Lens Utilization for Earth Surveillance}

Before worrying abut how to build and field large diffractive telescopes, we have to first address a more fundamental challenge; will they be optically useful at all? Diffractive optics (lenses or reflectors) focus different colors to different places; a given element is in-focus for only a single color at a time. For this reason, diffractive optics are generally used only for monochromatic, i.e., laser, applications. Such a limitation would still enable diffractive lenses to be valuable for laser weapons, or for imaging via active illumination, but not for traditional, passive, broadband imaging.

There is, fortunately, a way to get around this problem, and to build broad-band diffractive telescopes. The solution is to use a principle known as Schupmann correction, in which one dispersive element (in our case a diffractive optic) is used to correct the errors created by another one. The basic principle is shown below:

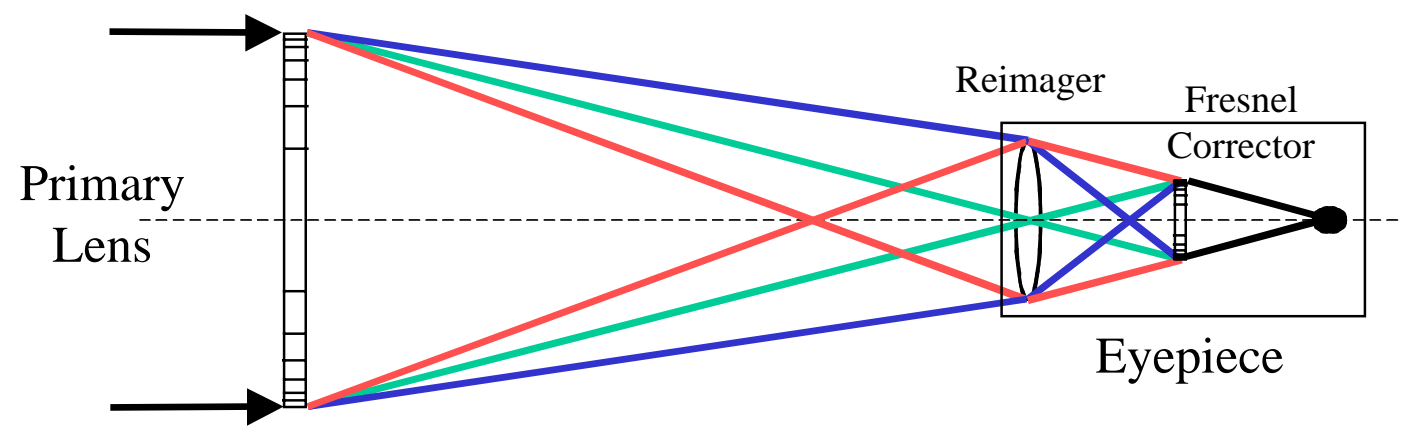

Broadband light enters the diffractive Primary Lens and is split apart; each color proceeding towards a separate focus. As it enters the telescope's Eyepiece, the light is intercepted and reimaged upon a second diffractive element, which spectrally recombines it; the resultant achromatic light is then brought to a common focus. The color-correction can be viewed as a two step process; a Reimager spatially recombines all light leaving a site on the Primary, and a Fresnel Corrector then angularly recombines it. Crucially, it is 
not necessary for the Fresnel Corrector to be the same size as the Primary Lens; the lens can be a large, 100 meter, thin-film device, while the Corrector can be a more rigid, 1 meter-sized element.

This color-correction process has been experimentally verified using a $50 \mathrm{~cm}$ aperture diffractive telescope; it achieved diffraction-limited focusing over a broad 470$700 \mathrm{~nm}$ spectral window. Optical designs have been made for even wider-bandwidth diffractive telescopes, capable of continuous spectral coverage for UV - MWIR wavelengths, with simultaneous LWIR coverage. So, diffractive telescopes can be used for broadband imaging, they are not only a monochromatic tool.

Use of large diffractive lenses for GEO-based Earth surveillance requires development of three additional types of systems (beyond the large lens). These are the secondary optics needed to collect and chromatically correct images, an active illumination system in order to collect images during night-time or cloudy conditions, and the auxiliary systems needed to operate the telescope.

\section{Passive Imaging}

The most straightforward way to perform earth surveillance using a large diffractive telescope is to use passive imaging, catching light emitted from target sites on the earth. This light can originate either from reflected sunlight (in the UV, visible, or NWIR), or from thermal emissions (MWIR or LWIR). In either event, light emitted from the target arrives at the large lens and is focused by it onto a separate Eyepiece vehicle, located several kilometers away. The Eyepiece is a space telescope in its own right; it has to receive the incoming light, spectrally recombine it, and form high-quality images.

The design of the Eyepiece optics is dominated by the need to do color-correction, converting the system from a micro-bandwidth $\left(\Delta \lambda / \lambda \sim 10^{-6}\right)$ instrument into a broadband telescope. As sketched above, two types of secondary optics are needed to perform this color-correction, a Reimager and a Fresnel Corrector. In order to deliver well-corrected, diffraction-limited images, each of these systems requires high-precision, meter-class optics.

The Reimager has two basic jobs, collecting a broad $\Delta \lambda$ band of the incoming spectrally diverging light, and precisely imaging the large diffractive lens onto the much smaller Fresnel Corrector.

In order to collect a broad spectral range of light, the Reimager must have sufficient aperture. This occurs because different colors spread apart as they leave the large diffractive lens. By the time they reach the distant Eyepiece, spectral width has been converted into a spatial width. In order to capture a given $\Delta \lambda / \lambda$ spectral window, the Reimager aperture, $D_{R}$, must be 
Because the Reimager size is proportional to the size of the large diffractive Primary Lens, it cannot be a small optic. A 2 meter Reimager is capable of only capturing a $4 \% \Delta \lambda / \lambda$ window from a 100 meter lens. Given the lens's $10,000 \mathrm{~m}^{2}$ collecting area, the concern about such a narrow window is not signal strength, but rather the restriction of information to such a narrow range of colors. Fortunately, as we'll discuss shortly, one can get around this limitation and collect simultaneous multi-band images; each spectral window has the same limited $\Delta \lambda / \lambda$ width, but together they cover a diverse range of wavelengths for use in multi-spectral image analysis.

In order for accurate color-correction to be possible, the Reimager must perform near-perfect, achromatic, mapping of points on the surface of the Primary Lens onto matching points on the Fresnel Corrector. Optically, the Reimager must be a finiteconjugate telescope; imaging between the two diffractive elements. In order to achieve the required achromatic performance, the Reimager should use reflective elements, not refractive ones. In order to deliver aberration-free performance, it should involve multiple elements, not the single element shown in the above schematic. Detailed optical designs of such Reimagers have already been performed by several teams for large 100 meterscale diffractive telescopes. While the Reimager requires complex, off-axis elements, they are well within the community's fabricational capabilities.

The second key component for color-correction is the Fresnel Corrector. Like the large Primary Lens, this is a diffractive element, however it is a much smaller (meterclass) optic, made from conventional, rigid optical materials. The challenge of the Fresnel Corrector, is that it must be much 'stronger' than the large lens, having more diffractive power. This occurs because the small Corrector must cancel-out the diffractive spreading of the much larger lens. In order to do so, its diameter and diffractive f-number are linked to those of the lens:

As with the Reimager, the fact that the Corrector's size is proportional to that of the lens, requires that it be a large optic. In order to keep the Corrector's size small, its fnumber, $f_{F}$, must also be small. Unfortunately, there is a firm limit on how low this can be; small f-numbers require small diffractive grooves, and these cannot function when smaller than the wavelength of light. If, considering also fabricational challenges, the Corrector's f-number is set at 1 , then matching a 100 meter lens to a 1 meter Corrector requires the lens's f-number to be 100; this demands a $10 \mathrm{~km}$ spacing between the two vehicles of the telescope. The color-correction process tightly couples the size of the two diffractive optics with their spacing; for large aperture telescopes it is an even stronger driver towards large separations than are lens-tolerance issues.

After being spectrally recombined by the Corrector, the light is diverging; in order to form images, it must be brought to a focus. Since the light is now achromatic, it can be 
focused with conventional, reflective optics. Considerable design freedom is possible here, but the simplest designs utilize a reflective Fresnel Corrector, merging its colorcorrection function with the refocusing one. Detailed optical designs exist for complete diffractive telescopes, in which reflective Fresnel Correctors work in concert with multielement Reimagers to provide broadband, diffraction-limited imaging.

Fabrication of the Fresnel Corrector will likely prove considerably more challenging than that of the Reimager elements. The basic element is of similar nature and complexity, it consists of an asymmetrically curved, meter-class, high-precision surface. The complication which makes it much harder to build, is that the Fresnel Corrector's surface must be covered with a precise pattern of $\mu \mathrm{m}$-sized grooves, in order to give it the required diffractive optical power. At present, the only diffractive optics of such aperture, groove-size, and precision are made on simple flat surfaces, not complex curved ones. There are, however, fabricational techniques that appear capable (with development) of patterning such surfaces; holographic and e-beam patterning are two promising approaches.

A color-corrected telescope such as described above, can, with very little effort, be enhanced to provide simultaneous coverage over multiple spectral windows. This is achieved by using the fact that diffractive optics can bend light into a discrete set of directions, not just one. With properly shaped grooves, a lens can be made to focus a harmonic set of colors to the same place. A lens with sawtooth-shaped grooves (with optical depth of $\lambda_{\bullet}$ ) will have the same focal point for wavelengths $\lambda_{\bullet}, \lambda_{\bullet} / 2, \lambda_{\odot} / 3, \lambda_{\odot} / 4$, and so on. Since the Reimager linking the two diffractive elements is achromatic, if both the Lens and the Corrector use such grooves, then the telescope will have color-corrected spectral windows centered around each of the harmonic wavelengths. As an example of such a system, suppose we set $\lambda$. to be $4 \mu \mathrm{m}$. The diffractive telescope will provide windows (each with $\Delta \lambda / \lambda \sim 4 \%$ ) at wavelengths of $4 \mu \mathrm{m}, 2 \mu \mathrm{m}, 1.33 \mu \mathrm{m}, 1 \mu \mathrm{m}, 0.8 \mu \mathrm{m}$, $0.67 \mu \mathrm{m}, 0.57 \mu \mathrm{m}, 0.50 \mu \mathrm{m}$, etc.

The 'price' of this multi-band capability is that the grooves must be 8-fold deeper and wider than if the telescope was only being used for visible $0.5 \mu \mathrm{m}$ light. The fact that the grooves are wider does not, unfortunately, make them easier to make; if we want to use them for $8^{\text {th }}$-harmonic, $0.5 \mu \mathrm{m}$, light, then they need the same precision as grooves would for direct $0.5 \mu \mathrm{m}$ operation. Multi-band lenses provide obvious benefits, but there is a penalty; deeper grooves require a thicker membrane than shallower grooves would, and hence lead to a heavier lens.

As with any large, high precision telescope, the secondary optics for a diffractive surveillance platform must be shaped and held-in-position to tight tolerances. The required fabricational precision can be met with the same methods and care as in traditional telescopes, particularly since standard, rigid materials can be used. The extra complication here, is that the Eyepiece's secondary optics must operate in cooperation with a distant, non-rigid, diffractive lens. Accordingly, they must be able to deal with both the effects of lens distortions and stationkeeping/alignment errors between the two spacecraft. 
Precision stationkeeping is, fortunately, not nearly as severe a challenge as might be anticipated. While the telescope must be aligned and positioned with high (few $\mu \mathrm{m}$ level) accuracy, the vehicles themselves do not have to be. Precision will be maintained with a dual-level control strategy. Here, the inter-vehicle positions and alignments will be controlled with a slow, coarse, outer-control loop. The telescope's optical precision is maintained by a much faster, tighter, inner-control loop, which tweaks the position and orientation of optical elements within the coarsely controlled Eyepiece vehicle. The division-of-labor between the two systems, i.e., the permissible coarseness on the vehicle's position and attitude, is determined by the system's optical aberrations; there is a limit beyond which simple rigid-body shifts of secondary optics is insufficient. The necessary tolerances have been examined for telescope point-designs; foot-scale lateral misalignments are tolerable, and multi-meter separation errors can be accommodated. The most sensitive error is a tilt one; the LOS of the secondary telescope must be accurately pointed towards the center of the lens. Optically, the effect of such misalignment is that the image of the lens is no longer centered on the Corrector, preventing accurate color-correction. The nature of the problem also leads to its methodof-control; as long as secondary optics are shifted slightly to keep the two diffractive elements centered on each other, LOS tilts can be cancelled out. In practice, the feedback loop is easily closed; a beacon can be placed in the center of the lens, the Reimager naturally projects this onto the surface of the Fresnel Corrector, and shifts are made to keep the spot on-center.

Simple tilts and shifts are capable of dealing with inter-vehicle stationkeeping/alignment errors. More complex actions are necessary to handle the effects of distortions within the lens itself. The loose tolerances of the diffractive lens greatly reduce the size of its optical distortions; but to deal with any that do occur, we need an adaptive optics system. As with conventional telescopes, one prefers to apply adaptive corrections to the small secondary optics, rather than the large primary. However, because in a diffractive telescope the secondary optics are responsible for color-correction, changes which improve the image at one wavelength may distort it at other wavelengths.

Two basic approaches can be utilized. The first is to exploit the reimaging nature of a color-corrected diffractive telescope, and to apply corrections directly at the Fresnel Corrector. The challenge here, is that this element is also responsible for color-correction. To deal with this, we will likely have to apply both piston and sideways displacements at the correction sites. The alternative method, is to apply corrections later in the optical chain, after light has been spectrally recombined. This will involve more complex static optics, but simpler adaptive ones.

\section{Active Imaging}

The lure of GEO-based surveillance platforms is that they are always in position', offering the potential to, if necessary, keep permanent watch over selected targets. Unfortunately, platforms which can only image in daytime and clear sky do not 
really deliver continuous coverage. Accordingly, extension of imaging to night-time and cloudy weather conditions (near-24/7 imaging) will greatly enhance the utility of GEObased diffractive-lens telescopes for earth surveillance. They will not only allow nearcontinuous coverage of chosen targets, but will also provide the ability to perform ondemand inspections of targets, watching them at (unpredictable) times of our choosing.

One night-time viewing approach is passive imaging, using MWIR or LWIR light. This can be done with the multi-band approach discussed in the previous section. Materials due exist (such as the Kapton, CP1, and TFDB polyimides) which are transparent for these IR wavelengths as well as for visible light. There are, however, two concerns with adopting this approach for night-time imaging. The first, is that grooves deep enough to allow MWIR or LWIR imaging lead to thicker, hence heavier, lenses than ones limited to visible or NWIR operation. The second, even greater, drawback to using MWIR/LWIR for night-time viewing, is that the images will be much lower resolution than visible ones; there is a 10-fold penalty for $5 \mu \mathrm{m}$ MWIR, and a 20 -fold one for $10 \mu \mathrm{m}$ LWIR.

In order to avoid this penalty, one can use active laser illumination for night-time viewing. Since efficient visible and NWIR lasers are available, this approach allows highresolution imaging throughout the night. Accompanying this major advantage, laser illumination does involve some drawbacks; the site's owner may learn that he is being watched, the platform needs a laser system, and (to reduce laser power) viewing is done by narrow-FOV rastering rather than wide-FOV snapshots.

In aiming for $24 / 7$ viewing capability, it is also essential to be able to image through clouds. While thick clouds present severe imaging problems, thin-to-moderate clouds are more common; laser illumination can be used to image targets through such clouds. Two basic methods appear attractive to reduce cloud-induced blurring; one uses time-of-flight, and the other uses angular-directionality. In the first approach, a very short pulse is sent, and the imaging sensor is time-gated, so as to only respond to unscattered, 'ballistic', photons. In the other method, a tight angular beam is transmitted, and sensors are gated to only respond to returning photons from precisely the same direction. Obviously, both methods can be combined to provide more selectivity, allowing penetration of thicker clouds.

The laser power required for active illumination is very much less than that needed for GEO-based weapons; their development will be discussed in that section, not here.

One obvious attraction of using laser illumination with a diffractive telescope, is that the light is monochromatic and thus does not have to be color-corrected. This raises the issue of whether all imaging should be done with lasers instead of passively. The advantage of the passive approach is that one gets multi-colored, multi-kilometer wide snapshots; the active approach eliminates the need for color-correction, allowing a smaller, more closely-spaced, secondary system. 


\section{Auxiliary Systems}

While the secondary optics are the Eyepiece 'payload' which makes an earth surveillance telescope function, the vehicle also needs a host of auxiliary support systems in order to operate. These systems include a power supply, propulsion, attitude control, imaging sensors, telemetry gear, a command system, etc. To a large extent, these auxiliary systems are similar to those on many other spacecraft, do not pose significant challenges, and will not require major development.

One crucial set of systems, are those which collect the incoming images and then transmit them down to earth. While pixel count and transmission bandwidth are major concerns for some applications, they are not for GEO-based diffractive telescopes. The reason for this, is that these long focal-length systems have a fairly narrow FOV; they take small pictures, not large ones. A typical image will be $\sim 10,000$ pixels across, and hence contain some 100 mega-pixels; this is not a troubling size for future systems. Accordingly, neither sensor (e.g., CCD) sizes or transmission rates should require specialized development for this application.

One system that will require dedicated development is moving the optics in order to look at desired targets. This steering requires relative motion between the two vehicles, so can be handled by either one; for practical reasons the propulsion system should probably reside on the secondary platform, not the lens one. Because the motion control requires large $\Delta \mathrm{Vs}$ but small accelerations, it will be performed with electrical, not chemical, propulsion. When analyzing the $\Delta \mathrm{V}$ requirements, one finds that they are dominated by the back-and-forth lateral moves needed to collect images, not by the steady radial thrust needed to 'tie' the two vehicles together.

For anticipated tasking scenarios, the propulsive cost is dominated by small, frequent, moves to look at nearby targets, not by the less frequent moves between distant sites. Accordingly, significant fuel savings can be realized by developing a fuel-less motion system to image nearby targets, moving the optics but not the vehicle's center-ofmass. This type of system, unique to this application, can be implemented in many different ways. One illustrative approach is to mount a flat turning mirror on a boom, move it to the appropriate image site, and bounce the incident light into the heavier, stationary, color-correction optics; a 25 meter boom, for example, allows rapid, fuel-less imaging throughout a $200 \mathrm{~km}$ ground region.

\section{Implementation}

In order to use a large diffractive lens for earth surveillance, we have to develop both the lens and the imaging platform. While the diffractive pattern used for a surveillance lens will likely differ from that used in a laser weapon, the overall lens development and implementation is essentially identical; we'll discuss lens development here, but it is also applicable to the laser weapon discussed in the next section of this report. This discussion will focus both upon what is needed to develop a large diffractive 
lens, and also what's needed to develop the imaging platform necessary for earth surveillance.

\section{Lens Technical Development}

\subsection{Years $59-63 \mathrm{M} \$$}

The lens development will last for 3.5 years and cost up to $63 \mathrm{M} \$$. At the end of this effort, we will have selected a lens material, and will have demonstrated a highthroughput method for forming the material into diffractive lens segments. A method for packaging and protecting the large lens during launch will have been developed and demonstrated through launch simulation. The configuration for the full-scale, spacebased lens will have been chosen, and the detailed design of its launch, deployment, and operation will be completed. Finally, the basic deployment, support, and precision characteristics of the lens will have been demonstrated by a large (30 meter) ground prototype.

The lens development program has four major thrusts; materials, patterning, launch packaging, and space support.

\section{Materials \\ $2-3$ Years \\ $6-9 \mathrm{M} \$$}

The goal here is to develop and produce the material for these thin-film lenses. The developed material must be transparent (for operational visible and IR wavelengths), be space-stable (acceptably-small property changes over 10 years in GEO), and have low thermal expansion (as far under $1 \mathrm{ppm} /{ }^{\circ} \mathrm{K}$ as possible). In addition to these intrinsic properties, the material must be producible in thin-films $\left(0.1 \mathrm{~kg} / \mathrm{m}^{2}\right.$ or less), in adequate quantities (at least $200 \mathrm{~m}^{2} / \mathrm{yr}$ production rate), and at affordable costs (at most 100 $\mathrm{K} \$ / \mathrm{m}^{2}$ ). In addition to being producible in adequate amounts, the material must be delivered in big enough pieces (at least 0.5 meters in size); while either panels or strips can be utilized, strips are preferable.

To be an acceptable lens material, we must also be able to pattern and package it. A material that makes either of these tasks impossible (or impracticably difficult) will not be acceptable; the easier a material is to pattern and package, the more preferable it is.

The goal during the first phase of effort will be to develop materials (both polymers and inorganics) with the right properties, and to demonstrate a process for manufacturing each in thin-film form; two separate teams will examine each option. The key issues for the polymer will be demonstrating low thermal expansion and spacestability; for the inorganics the emphasis is upon material toughness and the ability to make thin, uniform sheets.

The leading candidate for a polymer material is a polyimide based on the TFDB diamine. This type of polyimide was invented in Japan for semiconductor applications, and has been paired with a variety of different dianhydrides to make low CTE polyimides. The particularly interesting feature of this set of materials is that, depending upon the dianhydride chosen, the polyimide's CTE can be either low and negative or low 
and positive. These values are already much lower than other polyimides (e.g., Kapton, $\mathrm{CP} 1$, etc.); what makes them even more compelling for large diffractive lenses is the mixing (via blending or copolymerization) of a positive and a negative dianhydride to produce a polyimide having essentially zero thermal expansion. So, the goal during the first phase of this effort will be to formulate a zero-CTE polyimide.

The concerns with TFDB-based polyimides are that they are not commercially available in large thin films, and have never been space-tested yet. Production of large films is, of course, routinely done for other polyimides, so is not expected to present a challenge here. Films large enough for test purposes can be quickly made by spin-casting or doctor-blading. LLNL has previously made and had tested TFDB-based films in vacuum and cryogenic conditions (transparent lenses in space are cold), with good results. After a zero-CTE polyimide is formulated, small-film testing (optical, mechanical, vacuum, and space-radiation) will be performed determine its suitability for space-based lenses.

First phase development of inorganic films will concentrate upon the development of thin, non-brittle films. Improvements in toughness will be sought through choice of materials (nitrides as well as oxides) and configurations (e.g., multilayers). Growth-type methods such as CVD or sputtering will be examined for the production of thin (10-50 $\mu \mathrm{m})$ free-standing films. One of the challenges here, is that free-standing films are required. In principle, free-standing films can be attained by growth on a sacrificial substrate (e.g., silicon or high-temperature polymer) which can then be easily removed; in practice, the challenge is dealing with strains locked-in during growth, which are then released after the substrate is removed.

For the second phase of development, one material will be selected, and its manufacturing process will be scaled-up to the requisite unit-size and throughput. If polyimide materials are adopted, this scale-up is expected to be straightforward, as commercial roll-to-roll production of wide thin-films is routinely done for many polymers (including polyimides such as Kapton and CP1). Scale-up of inorganic film production will be more challenging, as there is currently no production of large-area, free-standing, thin-films. The reason for optimism, is that related capability does exist, for instance the production of large free-standing glass-sheets, and that of large sputteredcoatings. Unfortunately, neither existing process is directly applicable; the glass sheets are thick and brittle, while the sputtered films are thin coatings, not free-standing sheets. Scale-up will likely be risky (strain-release problems will get worse at larger sizes), and expensive.

\begin{tabular}{|c|c|c|}
\hline Phase I (do both) & 1 year & $4.0 \mathrm{M} \$$ \\
\hline Polymer (2 teams) & 1 year & $2.0 \mathrm{M} \$$ \\
\hline Inorganic (2 teams) & 1 year & $2.0 \mathrm{M} \$$ \\
\hline & & \\
\hline Phase II (do one) & $1-2$ years & $2.0-5.0 \mathrm{M} \$$ \\
\hline Polymer & 1 year & $2.0 \mathrm{M} \$$ \\
\hline Inorganic & 2 years & $5.0 \mathrm{M} \$$ \\
\hline
\end{tabular}


The goal here is to convert the thin-film material into sections of a diffractive lens. This requires both smoothing and patterning. In smoothing, the film's optical thickness is measured, and then it is sculpted accordingly, to a spatially constant value. Next, the film is diffractively patterned by cutting shallow, narrow grooves into its surface.

This development will proceed in three phases. The first phase will demonstrate whether-or-not each of the lens materials being developed above can be effectively patterned. This task must be completed before the material down-select is made. The next phase of development is to scale-up patterning of the chosen material to full-size pieces (strips or panels). This work will concentrate upon size, throughput, and local-precision; it will also demonstrate local-smoothing. The final phase of this effort will be to achieve global precision, both in smoothing the film to a constant thickness, and in the positioning of the surface grooves.

The methods used for these tasks differ, of course, depending upon the material chosen for the lens. For polymers, both laser ablation and embossing-type methods will be evaluated during the first phase. Both processes are commercially used to pattern polymers, and application of diffractive groove patterns has been demonstrated. For laser ablation, the key thing to determine in this phase is the effect of debris from ablated material, for embossing/extrusion techniques the issue is how well (and rapidly) hightemperature polyimides can be patterned. For inorganics, lithography is a very well established technique for application of diffractive patterns; the only issue here is to characterize etching of the particular materials selected.

Second phase patterning scale-up will be performed on only one material, the polymer or inorganic selected as the lens material. If a polymer is chosen, then we must also decide whether to use laser ablation or embossing/extrusion for full-scale patterning. This selection will draw upon the first phase results, specifically whether debris is a major problem in laser ablation and whether precision appears attainable in embossing or extrusion. Scale-up of either process for the roll-to-roll patterning of full-size $(0.5-1.5$ meter) strips should be straightforward, as modestly-customized commercial machines can be used. A smoothing process must also be demonstrated; this will be attempted for laser ablation via the closed-loop, mask-based process discussed previously, and for embossing/extrusion by precision control of the deformation.

If an inorganic material is selected for the lens material, then large-size patterning should also be straightforward to achieve. If high-toughness material can't be developed, then an inorganic-based lens would be best-assembled in-space from separate panels; lithography is already capable of patterning meter-sized panels (72 such panels were made and used in LLNL's 5 meter lens). If a sufficiently tough material is developed, then strip-based lenses (e.g., the Square Ribbon design) will likely be adopted, so a rollto-roll patterning approach is required. Adaptation of lithography for this process will be 
straightforward, since linear resist-coating, exposure, wet-etching, and resist-stripping processes have already been developed (and combined) at LLNL. It is possible (and highly desired) that the processes used to make the thin sheets will directly deliver the necessary $(100 \mathrm{~nm})$ thickness uniformity; in this case, no additional smoothing will be required. In the event that this direct smoothing is not achievable, then a smoothing process must be demonstrated during this phase. Mechanical polishing is, of course, routinely used for thick materials; recently high-throughput, feedback-controlled MRF techniques have been demonstrated for thinner, mm-class, sheets. If this can be used on the more delicate $10-50 \mu \mathrm{m}$ films needed here, then mechanical polishing will be an attractive smoothing approach. An alternate, lower force, approach has also been recently developed; this exploits the strong thermal etch-rate dependence in standard, wet-etching of inorganics. Here, an array of independently-controlled heat sources is used to apply a feedback-controlled spatial temperature distribution to a uniform liquid etchant; real-time measurements of the film's spatial-profile are made during etching, and the thermal profile is adjusted accordingly to deliver a uniform film. This process has been demonstrated at 18 inch size with a 64 x 64 grid of switchable (PCB mounted) resistors; it can be readily adapted for use in a roll-to-roll process.

The final phase of pattering development is to demonstrate global precision of the smoothed and patterned lens material. While the inability to achieve global precision will not prevent the fielding of 100 meter-scale diffractive lenses (since adaptive optics can be used to compensate lens errors), it remains highly desirable. For smoothing, the step from local to global precision should be 'free', since local thickness control will be achieved by smoothing to a set target value. Patterning presents more challenges, since the locallyprecise grooves must be kept globally straight over full length of the strips.

The effort required to achieve global precision will depend on the material and patterning methods adopted. If polymers are chosen, then laser ablation offers the cleanest route to global precision, since it involves no physical contact or bulk heating. Here, a straight reference line can mapped onto the material (relative to its border or other marks), and the laser-ablation pattern applied relative to this line. For inorganics, no global patterning is required if on-orbit panel assembly will be used; if long strips are made, then a process similar to that above can be used.

\begin{tabular}{|c|c|c|}
\hline Phase I (do both) & 1 year & $2.0 \mathrm{M} \$$ \\
\hline Polymer & 1 year & $1.0 \mathrm{M} \$$ \\
\hline Inorganic & 1 year & $1.0 \mathrm{M} \$$ \\
\hline Phase II (do one) & & \\
\hline Polymer & 1 year & $2.5-3.5 \mathrm{M} \$$ \\
\hline Inorganic & 1 year & $3.5 \mathrm{M} \$$ \\
\hline & 1 year & $2.5 \mathrm{M} \$$ \\
\hline Phase III (do one) & 1 year & $1.5 \mathrm{M} \$$ \\
\hline Polymer & 1 year & $1.5 \mathrm{M} \$$ \\
\hline Inorganic & 1 year & $1.5 \mathrm{M} \$$ \\
\hline
\end{tabular}




\section{Packaging}

2.5 Years

$7-9 \mathrm{M \$}$

The goal here is to develop the method by which the thin-film lens material is packaged for launch and then deployed in space. This task is interrelated to the other developmental efforts as well, since it depends on the material chosen, its format (strips or panels), and the support method adopted (rotation, truss, external ring).

This work will be split into a design phase and a prototyping phase. The design effort will concentrate upon two different packaging approaches, the use of rolled-up strips, and that of separated panels; each examined by two separate teams. Two other methods, monolithic-folding and fold-then-roll will be considered, but currently appear more difficult. The design emphasis for the strip approach is upon the roll used to hold the strips; this roll-holder must protect the strip during launch, and then smoothly and reliably deploy it in space. For use of separate panels, the key challenges are to design a stack-holder for packaging and protecting the panels during launch, and an alignment/assembly robot which puts the panels together in space.

After this set of packaging options is examined during the design phase, one will be selected and carried forward in the second, prototyping phase of development. The approach which is selected depends both on the packaging merits and feasibility determined in the design phase, and on the parallel selection decisions made regarding the lens material, format, and support structure. The goal of this phase will be to build and demonstrate the packaging hardware; the launch packaging system will undergo shake-and-vibration testing, and the deployment system will be tested (strips will be unrolled, or panels will be unstacked and joined together).

\begin{tabular}{|c|c|c|}
\hline Phase I (do both) & 1 year & $4.0 \mathrm{M} \$$ \\
\hline Rolled Strips & 1 year & $2.0 \mathrm{M} \$$ \\
\hline Stacked Panels & 1 year & $2.0 \mathrm{M} \$$ \\
\hline & & \\
\hline Phase II (do one) & 1.5 years & $3.0-5.0 \mathrm{M} \$$ \\
\hline Rolled Strips & 1.5 years & $3.0 \mathrm{M} \$$ \\
\hline Stacked Panels & 1.5 years & $5.0 \mathrm{M} \$$ \\
\hline
\end{tabular}

\section{Structural Support}

3.5 Years

$39 \mathrm{M} \$$

The goal here is to determine what type of lens configuration should be used, to design the structural and operational systems needed to support the lens, and then to build and demonstrate a 30 meter ground-prototype of the lens. This effort is clearly strongly related to the other developmental ones, it influences and is influenced by the choice of lens material, material format, and packaging methods.

This development will proceed in three phases, two concentrating on design, and one on building and demonstrating the lens prototype. The first design phase is a preliminary one; its purpose is to examine various types of lens configurations (e.g., 
Circular Rotating, Square Ribbon, etc.), and allow one to be selected for further development. This effort will involve multiple separate teams (nominally 3 ), which will examine different types of lenses with regard to issues such as deployment, mass, vibrations, attitude and motion control, lens distortions, flight-readiness, etc. At the end of this phase (and in consideration of results from the other developmental efforts) one lens configuration will be selected for further development.

The second and third phases of this development effort will proceed in parallel. One portion (phase II) is focused upon the detailed design of support systems for a fullscale, space-based lens; the other effort (phase III) is aimed at building a 30 meter ground-prototype of the lens. The Phase II design effort is more detailed than the preliminary one in Phase I, performing in-depth analysis of the lens and its support systems. The Phase III prototyping effort is intended to build, and demonstrate on the ground, a 30 meter prototype of the diffractive lens. While ground-based deployment and operation cannot perfectly reproduce all aspects of a space-based unit, this prototype will demonstrate the basic functionality. It will start from a stowed configuration, proceed through deployment, and then structural lens support. If a separate panel approach is adopted, the prototype will include panel alignment and assembly; if a Square Ribbon design is used, the strips will be unrolled, and active control of strip locations will be demonstrated.

\begin{tabular}{|c|c|c|}
\hline Phase I (3 teams) & 1 year & $9.0 \mathrm{M} \$$ \\
\hline Preliminary Design & 1 year & $3.0 \mathrm{M} \$$ \\
\hline Phase II & & \\
\hline Detailed Design & 2.5 years & $10.0 \mathrm{M} \$$ \\
\hline & 2.5 years & $10.0 \mathrm{M} \$$ \\
\hline Phase III & & \\
\hline Prototype & 2.5 years & $20.0 \mathrm{M} \$$ \\
\hline
\end{tabular}

Development Schedule: Diffractive Lens

Materials

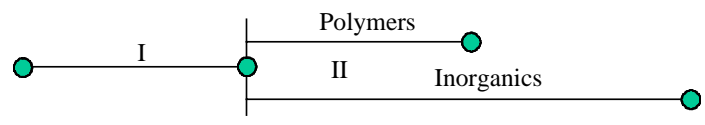

Patterning

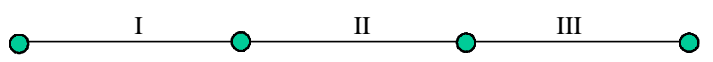

Packaging

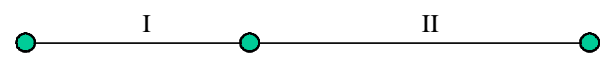

Support
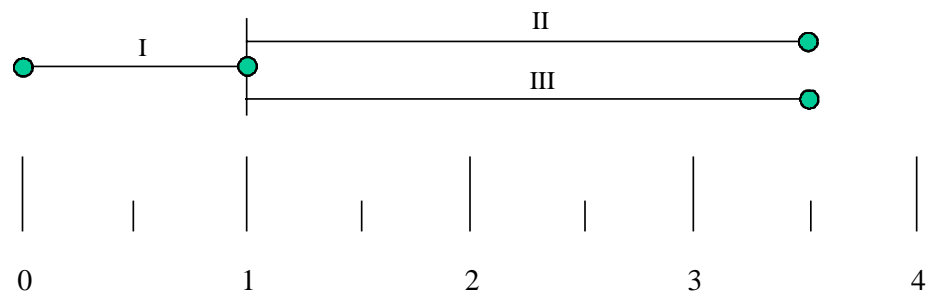


\section{Technical Development of Eyepiece $\quad 3.5$ Years $\quad 48 \mathrm{M} \$$}

In order to use a large diffractive lens for an earth surveillance platform, we also have to develop a mobile Eyepiece; this is the spacecraft which moves along the lens's focal surface collecting target images. The Eyepiece development for earth surveillance will last for 3.5 years and cost $48 \mathrm{M} \$$. At the end of this effort, we will have designed the Eyepiece vehicle (and prototyped novel sub-systems) in sufficient detail to permit the production, launch, and operation of a flight system. The Eyepiece's optical system will be fully designed, and will be ground-prototyped at full-scale. This system will be tested (with a diffractive test-plate in lieu of the 100 meter diffractive lens) to demonstrate its optical performance and sensitivities.

This development program has two major elements; the Eyepiece's optical payload, and the spacecraft systems that support this payload.

\section{Optical Payload \\ 3.5 Years \\ $25 \mathrm{M \$}$}

The goal here is to design, build, and test the Eyepiece optics that collect images from the large diffractive lens. This effort has three sub-tasks, optical design, fabrication of the Fresnel Corrector, and assembly and test of a full-scale prototype optical system. The development of the Eyepiece optics can proceed in parallel with development of the large diffractive lens, it does not have to wait for this work to be completed.

The design of the Eyepiece optics is a two phase process spanning three years. During the first year of effort, the design is general, as the diffractive lens development (proceeding in parallel) has not yet settled on a specific lens design. However, during this phase of design, the basic characteristics of the Eyepiece optics will be determined in a two team competition. The size, number of elements, and layout of the Reimager optics will be decided upon. Similarly, the size, basic shape, and diffractive power of the Fresnel Corrector will be determined; as will the nature of the post-Corrector optical chain. These optical systems will be designed together, in order to maximize optical performance (resolution, field-of-view, throughput, spectral band-width), while minimizing the size (mass and length as well as aperture) of the required optics. Preliminary consideration will also be given to the incorporation of adaptive optics into this system; the decision will be made whether adaptive optics will be incorporated into the Fresnel Corrector, or placed later in the optical chain.

After the lens design is chosen (circular or square layout, radial or linear grooves), the design of Eyepiece optics becomes more detailed. The shapes and tolerances of the optical elements are set, as are the mounting and support fixtures. Multi-spectral sensor arrays are designed to capture the images. Image correction systems are finalized, using shift/tilt adjustments to handle stationkeeping errors, and more complex adaptive optics to deal with lens distortions. The design phase overlaps the fabrication of a full-scale prototype Eyepiece telescope, so is also tightly coupled with the specifications and realities of this implementation. 
The second task of the Eyepiece optical development effort, is the fabrication of the Fresnel Corrector. This diffractive element is crucial in canceling out the large chromatic dispersion of the large diffractive lens, enabling the overall optical platform to deliver high-precision, broad-band images. Although much smaller than the primary lens ( 1 meter vs. 100 meters), the Fresnel Corrector is difficult to make; its diffractive grooves are much smaller ( $1 \mu \mathrm{m})$, and must be precisely located (to sub- $\mu \mathrm{m}$ precision) upon a highly curved surface. This fabrication challenge will be pursued by a 3 year, two phase, development effort. The first phase of this task will involve two teams, and determine the most appropriate fabrication method; leading candidates are electron-beam lithography and in-situ holographic patterning. After a basic fabrication route is determined, then the second phase of the effort will make a full-scale Fresnel Corrector for use in the prototype Eyepiece telescope.

The third development task is to build and test a prototype Eyepiece optical system. This is not intended to be a final, flight-ready, instrument, but will be a full-scale test-bed; enabling the optical performance of the diffractive telescope to be studied and verified on the ground. Obviously, the experience gained in assembling and testing a full system (Reimager, Fresnel Corrector, Sensors, and Adaptive Optics) on the ground before having to field a space-based flight version will be invaluable. What makes this development even more significant, is that it can be tested alone, without having to (somehow) combine it with a 100 meter diffractive lens located $10 \mathrm{kms}$ away. Accurate solo testing of the Eyepiece optics is possible because the incoming beam from a large, distant diffractive lens can be mimicked by a small ( 2 meter-sized) diffractive test-plate, mounted near the entrance of the Eyepiece optics; the test-plate is designed to deliver precisely the same multi-spectral, multi-focused beam as would a large distant lens. Accordingly, we can evaluate the optical performance and characteristics of the sophisticated Eyepiece optics on the ground (at full-scale), before having to field a spacebased system. Later, of course, the same test configuration can be used to verify performance of the actual, flight-ready system before it is launched.

\begin{tabular}{|c|c|c|}
\hline Optical Design & 3 years & $5.0 \mathrm{M} \$$ \\
\hline Phase I (2 teams) & 1 year & $2.0 \mathrm{M} \$$ \\
\hline Phase II & 2 years & $3.0 \mathrm{M} \$$ \\
\hline & & $11.0 \mathrm{M} \$$ \\
\hline Fresnel Corrector & 3 years & $6.0 \mathrm{M} \$$ \\
\hline Phase I (2 teams) & 1.5 years & $5.0 \mathrm{M} \$$ \\
\hline Phase II & 1.5 years & \\
\hline & & $9.0 \mathrm{M} \$$ \\
\hline Prototype System & 2 years & $9.0 \mathrm{M} \$$ \\
\hline Fabrication \& Test & 2 years &
\end{tabular}

Vehicle Systems

3 Years

$23 \mathrm{M} \$$

The goal here is to design the systems on the Eyepiece spacecraft which are needed to support the optical payload. For the most part this is a design effort, adapting 
already developed technical systems for use in this application. One significant development task is needed however; an image capturing system will be prototyped to allow fuel-less mobility in the acquisition of images from nearby ground targets.

The primary task here is the design of the non-payload portion of the Eyepiece spacecraft. This work will proceed in two phases. The first effort will use two competing teams to consider a range of system options and develop a basic vehicle design. This phase will consider both system and technology issues. The systems design will investigate the operational use of a GEO-based surveillance platform, including tasking, retargeting, image quality issues. The design effort will also consider a number of technology issues, including power-supply, propulsion, attitude control, thermal control, image transmission, lens-stationkeeping, etc. The teams will determine mass and cost estimates for the Eyepiece, as well as launch options for delivery of both the Eyepiece and the large lens to GEO. After this preliminary design phase is completed, a single team will be selected to perform a more involved vehicle design, sufficiently detailed to enable the production and fielding of a flight vehicle.

One sub-system of the Eyepiece is sufficiently novel as to require dedicated design and prototyping. This is a Mobile Image Capturing system, which enables images from nearby ground targets to be acquired rapidly and without fuel expenditure. During the first phase of this effort (in parallel with the first phase of the overall vehicle design), two teams will determine the best technical ways to accomplish this task. Then, during the second phase, one method will be selected and prototyped.

\begin{tabular}{|c|c|c|}
\hline Vehicle Design & 3 years & $16.0 \mathrm{M} \$$ \\
\hline Phase I (2 teams) & 1.5 years & $8.0 \mathrm{M} \$$ \\
\hline Phase II & 1.5 years & $8.0 \mathrm{M} \$$ \\
\hline & & \\
\hline Mobile Image Capture & 2.5 years & $7.0 \mathrm{M} \$$ \\
\hline Phase I (2 teams) & 1 year & $2.0 \mathrm{M} \$$ \\
\hline Phase II & 1.5 years & $5.0 \mathrm{M} \$$ \\
\hline
\end{tabular}




\section{Development Schedule: Eyepiece}

Optical Design

Fresnel Corrector

Eyepiece Fab \& Test

Vehicle Design

Mobile Image Capture
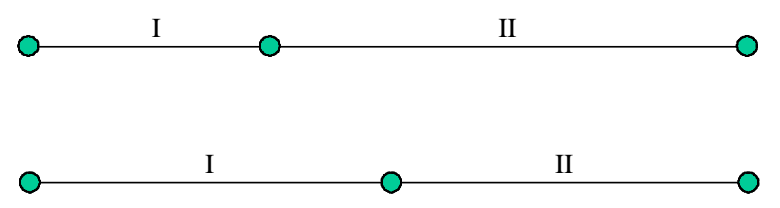

I
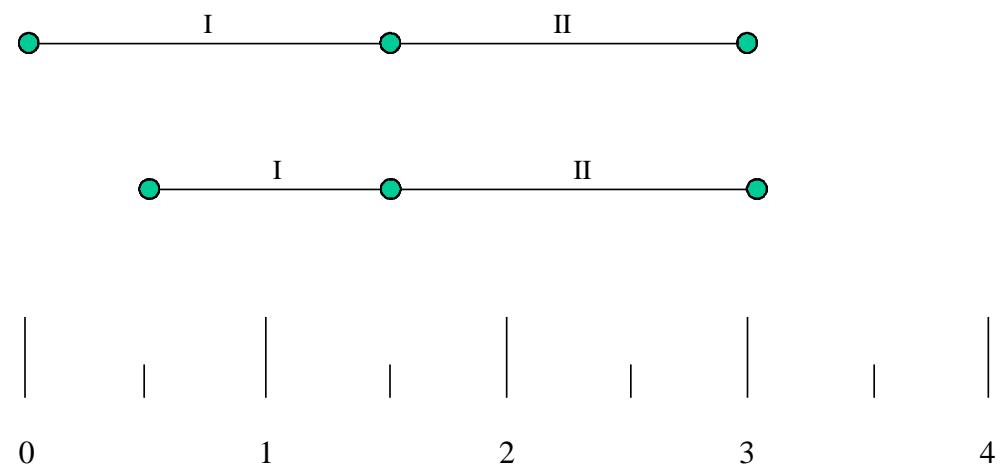

\section{Surveillance Implementation $\quad 3-8$ Years $\quad 680-780 \mathrm{M} \$$}

The implementation of this earth surveillance platform can commence as soon as the lens and Eyepiece technical developments are complete. Because the lens material will be produced, patterned, and packaged in sections (most likely rolled-up strips) its production is a self-contained process. Fabrication of other systems (e.g., the lens supporting structure and the imaging Eyepiece) can proceed independently. The surveillance platform will be launched 3 years after the implementation phase begins, 6.5 years after commencement of the overall program

The cost to field the surveillance platform will depend critically upon that of delivering it up to GEO. This cost, of course, depends on the mass of the system, the type and number of boosters needed for GEO delivery, and what booster costs will be at the time. While none of these things are known now, our current estimates are a $15-20$ metric ton mass, $2-3$ heavy EELVs, and a launch cost of $200-400 \mathrm{M} \$$.

The platform's mass is dominated by four systems, the diffractive lens, its structural support, the imaging Eyepiece, and fuel for imaging and stationkeeping. 


\begin{tabular}{|c|c|}
\hline System & Mass (metric tons) \\
\hline & \\
\hline Lens Membrane & 1 \\
\hline Lens Structure & 4 \\
\hline Eyepiece & 4 \\
\hline Fuel & 8 \\
\hline Total & 17 \\
\hline
\end{tabular}

The delivery of this platform to GEO depends upon how LEO-to-GEO transport is handled, and how the platform systems are grouped together for transport. We'll assume use of a large EELV for launch from the earth; this is typified by an Atlas V with 20 ton capacity into LEO and 8.5 for GTO. The straightforward GEO delivery approach (using the EELV for GTO insertion and then solid-fuel engines for braking into GEO) only delivers 3.5-4.0 tons, and would require 5 launches. A much preferable technique would be to use the EELV for GTO insertion, but to use electric propulsion for circularization into GEO; if carefully apportioned, the platform could be delivered with 3 EELV launches. One vehicle would carry the lens, one would carry the Eyepiece, and the last would deliver most of the fuel (some fuel would likely travel on the first two vehicles as well). A more aggressive delivery approach is use the EELVs only for delivery into LEO; using more fuel efficient propulsion for the LEO-to-GEO transport. This might be based upon other government-developed transfer stages or upon the platform's own electric array and propulsion capability. Use of such transfer options could reduce the number of EELVs required to two. The launch choice will depend upon the future cost of EELVs, and the availability of transfer propulsion. We'll budget $300-400 \mathrm{M} \$$ for GEO delivery, based upon either 3 EELVs at a cost of $100 \mathrm{M} \$$ each, or 2 EELVs at a higher cost of $200 \mathrm{M} \$$. If future EELV costs are in the $200 \mathrm{M} \$$ regime, then it is likely that 2 EELVs with alternative LEO-to-GEO transfer would be preferable to paying $600 \mathrm{M} \$$ for 3 EELV launches.

The cost of actually operating the surveillance platform is governed by the ground personnel required to monitor and operate the platform. We will budget $60 \mathrm{M} \$$ per year for a duration of 5 years. While the platform itself will last longer than this, it seems likely that technical advances will dictate its replacement by an improved, second generation, platform after 5 years of operation; even if not retired, the system will require refueling after this period.

\begin{tabular}{|l|c|c|}
\hline Payload Assembly \& Integration & 3 years & $80 \mathrm{M} \$$ \\
\hline Delivery to GEO & & $300-400 \mathrm{M} \$$ \\
\hline & & \\
\hline Surveillance Operations & 5 years & $300 \mathrm{M} \$$ \\
\hline
\end{tabular}




\section{Operational Schedule: Earth Surveillance}

Diffractive Lens

Eyepiece Vehicle

Integration \& Launch

Platform Operation
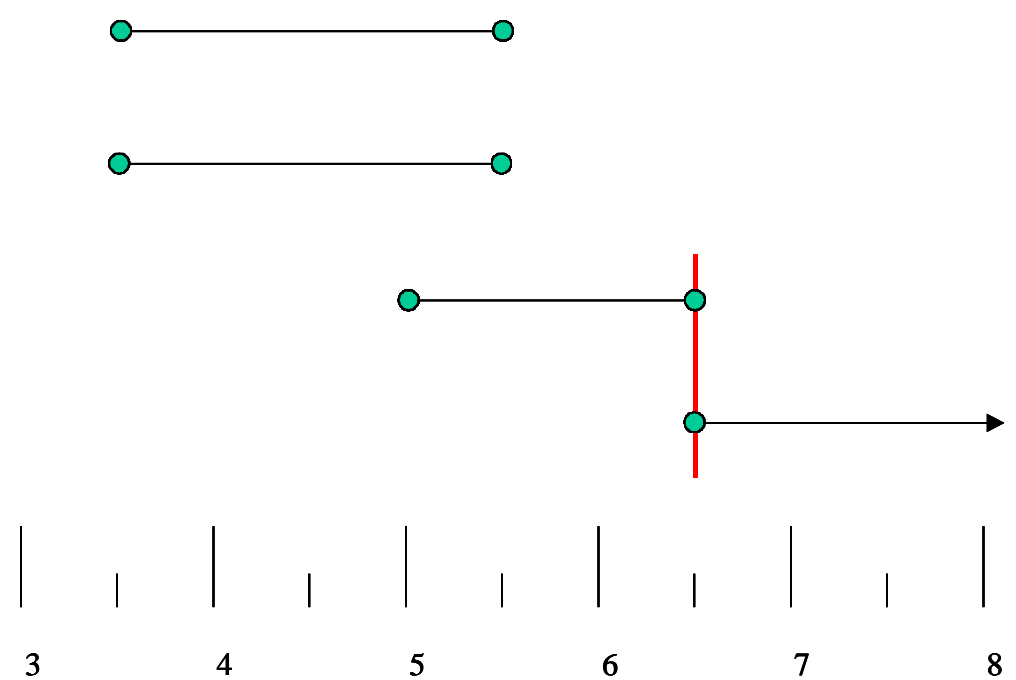

3

$\begin{array}{ll}\mid & 1 \\ 5 & 6\end{array}$

|

6

8 